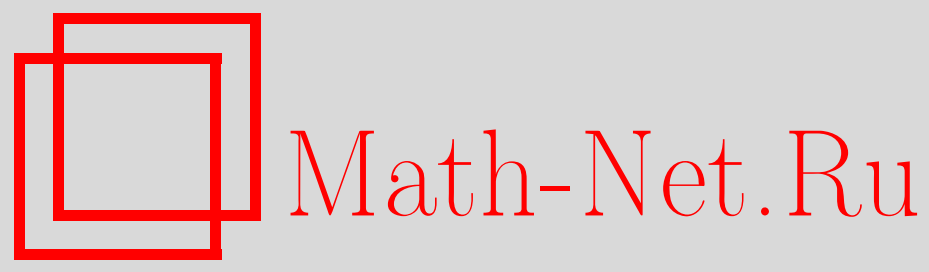

С. М. Гусейн-Заде, И. Луенго, А. МельеЭрнандез, Дзета-функции ростков мероморфных функций и диаграмма Ньютона, Функи. анализ и его прил., 1998, том 32, выпуск 2, 26-35

DOI: https://doi.org/10.4213/faa349

Использование Общероссийского математического портала MathNet.Ru подразумевает, что вы прочитали и согласны с пользовательским соглашением http://www . mathnet.ru/rus/agreement

Параметры загрузки:

IP: 34.227 .88 .159

26 апреля 2023 г., 15:42:00

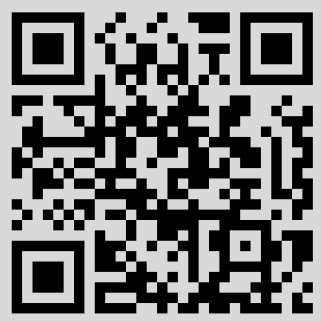


Функииональный анализ и его приложения

1998, т. 32, вып. 2, с. 26-35

УДК 517.9

\section{Дзета-функции ростков мероморфных функций и диаграмма Ньютона ${ }^{\star}$}

(C) 1998. С. М. ГУСЕЙН-ЗАДЕ, И. ЛУЕНГО, А. МЕЛЬЕ-ЭРНАНДЕЗ

\section{§1. Ростки мероморфных функций}

Многочлен $f$ степени $d$ от $n+1$ комплексных переменных определяет мероморфную функцию $f$ на проективном пространстве $\mathbb{C P}^{n+1}$. Чтобы понять поведение мероморфной функции $f$ на бесконечности, естественно рассмотреть ее ростки в точках бесконечно удаленной гиперплоскости $\mathbb{C P}_{\infty}^{n} \subset$ $\mathbb{C P}^{n+1}$. В локальной аналитической системе координат $z_{0}, z_{1}, \ldots, z_{n}$ с началом в точке $p \in \mathbb{C P}_{\infty}^{n}$ и такой, что бесконечно удаленная гиперплоскость $\mathbb{C P}_{\infty}^{n}$ задается уравнением $\left\{z_{0}=0\right\}$, росток функции $f$ имеет вид $f=$ $P\left(z_{0}, \ldots, z_{n}\right) / z_{0}^{d}$. Рассмотрим ростки мероморфных функций общего вида.

ОПРЕДЕЛЕНИЕ 1. Росток мероморфной функции на $\left(\mathbb{C}^{n+1}, 0\right)-$ это дробь $f=P / Q$, где $P$ и $Q$ - ростки голоморфных функций $\left(\mathbb{C}^{n+1}, 0\right) \rightarrow(\mathbb{C}, 0)$. Два ростка мероморфных функций $f=P / Q$ и $f^{\prime}=P^{\prime} / Q^{\prime}$ равны, если существует такой росток голоморфной функции $U:\left(\mathbb{C}^{n+1}, 0\right) \rightarrow \mathbb{C}$, что $U(0) \neq 0$, $P^{\prime}=U \cdot P$ и $Q^{\prime}=U \cdot Q$.

ЗАмечАния. 1. Для удобства мы не рассматриваем здесь функции вида $1 / Q(z)$ или $P(z) / 1$.

2 . В соответствии с определением $x / y \neq x^{2} / x y$, но $x / y=x \exp (x) / y \exp (x)$.

Недавно В. И. Арнольдом была дана классификация простых ростков мероморфных функций для некоторых отношений эквивалентности [2].

В дальнейшем мы будем систематически использовать разрешения ростков мероморфных функций.

ОПРЕДЕЛЕНИЕ 2. Разрешением ростка $f$ называется модификация пространства $\left(\mathbb{C}^{n+1}, 0\right)$ (т. е. собственное аналитическое отображение $\pi: \mathscr{X} \rightarrow \mathscr{U}$ неособого аналитического многообразия $\mathscr{X}$ на окрестность $\mathscr{U}$ начала координат в $\mathbb{C}^{n+1}$, которое является изоморфизмом вне собственного аналитического подпространства в $\mathscr{U})$, такая, что полный прообраз $\pi^{-1}(H)$ гиперповерхности $H=\{P=0\} \cup\{Q=0\}$ является дивизором с нормальными пересечениями в любой точке многообразия $\mathscr{X}$.

Тот факт, что $\pi^{-1}(H)$ является дивизором с нормальными пересечениями, означает, что в окрестности любой его точки существует такая локаль-

* Работа первого автора частично поддержана грантом РФФИ № 98-01-00612 и грантом INTAS-4373; работа последних двух авторов частично поддержана грантом CAICYT PB94-291. 
ная система координат $y_{0}, y_{1}, \ldots, y_{n}$, что поднятия $\widetilde{P}=P \circ \pi$ и $\widetilde{Q}=Q \circ \pi$ функций $P$ и $Q$ на пространство $\mathscr{X}$ разрешения равны $u y_{0}^{k_{0}} y_{1}^{k_{1}} \cdot \ldots \cdot y_{n}^{k_{n}}$ и $v y_{0}^{l_{0}} y_{1}^{l_{1}} \cdot \ldots \cdot y_{n}^{l_{n}}$ соответственно, где $u(0) \neq 0$ и $v(0) \neq 0, k_{i}$ и $l_{i}$ неотрицательны.

Пусть $B_{\varepsilon}$ - замкнутый шар радиуса $\varepsilon$ с центром в начале координат в $\mathbb{C}^{n+1}$, где $\varepsilon$ достаточно мало, так что представители ростков функций $P$ и $Q$ определены в $B_{\varepsilon}$ и для любого положительного $\varepsilon^{\prime}<\varepsilon$ cфера $S_{\varepsilon^{\prime}}=\partial B_{\varepsilon^{\prime}}$ пересекает аналитические пространства $\{P=0\},\{Q=0\}$ и $\{P=Q=0\}$ трансверсально (в стратифицированном смысле). Выберем достаточно маленькое $\delta>0$ и рассмотрим шар $B_{\delta} \subset \mathbb{C}^{2}$ радиуса $\delta$ с центром в начале координат.

ОПРЕДЕЛЕНИЕ 3. 0-слоем Милнора ростка $f$ называется множество

$$
\mathscr{M}_{f}^{0}=\left\{z \in B_{\varepsilon}:(P(z), Q(z)) \in B_{\delta} \subset \mathbb{C}^{2}, f(z)=P(z) / Q(z)=c\right\}
$$

для ненулевого $c \in \mathbb{C}$ с достаточно малым модулем $\|c\|$. Точно так же $\infty$-слоем Милнора ростка $f$ называется множество

$$
\mathscr{M}_{f}^{\infty}=\left\{z \in B_{\varepsilon}:(P(z), Q(z)) \in B_{\delta} \subset \mathbb{C}^{2}, f(z)=P(z) / Q(z)=c\right\}
$$

для $c \in \mathbb{C}$ с достаточно большим модулем $\|c\|$.

ЛЕмма 1. Понятие 0- (соответственно $\infty$-)слоя Милнора корректно определено, т.е. для достаточно малого $\|c\|, 0<\|c\| \ll \delta \ll \varepsilon$ (соответ-

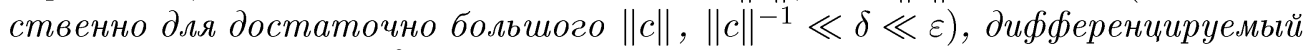
mип многообразия $\mathscr{M}_{f}^{0}$ (соответственно многообразия $\mathscr{M}_{f}^{\infty}$ ) не зависит om $\varepsilon, \delta u c$.

ДокАЗАтЕльство. Пусть $\pi: \mathscr{X} \rightarrow \mathscr{U}$ - разрешение ростка $f$, которое является изоморфизмом вне гиперповерхности $H=\{P=0\} \cup\{Q=0\}$, $r: \mathbb{C}^{n+1} \rightarrow \mathbb{R}$ — функция $r(z)=\|z\|^{2}$ и $\tilde{r}=r \circ \pi: \mathscr{X} \rightarrow \mathbb{R}$ - поднятие функции $r$ на пространство разрешения $\mathscr{X}$. Для достаточно малого $\varepsilon>0$ гиперповерхность $\widetilde{S}_{\varepsilon}=\left\{\tilde{r}=\varepsilon^{2}\right\}$ (прообраз среры $S_{\varepsilon} \subset \mathbb{C}^{n+1}$ ) трансверсальна ко всем стратам полного прообраза $\pi^{-1}(H)$ гиперповерхности $H$. В каждой точке из $\pi^{-1}(H)$ в локальной системе координат имеют место равенства $P \circ \pi=u y_{0}^{k_{0}} \cdot \ldots \cdot y_{n}^{k_{n}}, Q \circ \pi=v y_{0}^{l_{0}} \cdot \ldots \cdot y_{n}^{l_{n}}$ с $u(0) \neq 0, v(0) \neq 0$. Таким образом, $f \circ \pi=w y_{0}^{m_{0}} \cdot \ldots \cdot y_{n}^{m_{n}}$ с $w(0) \neq 0$. Вещественная гиперповерхность $\widetilde{S}_{\varepsilon}$ трансверсальна ко всем координатным подпространствам (различных размерностей). Нетрудно показать, что отсюда вытекает трансверсальность гиперповерхности $\widetilde{S}_{\varepsilon}$ к (комплексным) гиперповерхностям $\left\{w y_{0}^{m_{0}} \cdot \ldots \cdot y_{n}^{m_{n}}=c\right\}$ для достаточно малого $\|c\| \neq 0$ и для достаточно большого $\|c\|$. Теперь доказательство следует из стандартных аргументов.

ЗАмЕчАНИЯ. 1. Определение означает, что $\mathscr{M}_{f}^{0}$ или $\mathscr{M}_{f}^{\infty}$ равно

$$
\left\{z \in B_{\varepsilon}:(P(z), Q(z)) \in B_{\delta} \subset \mathbb{C}^{2}, P(z)=c Q(z), P(z) \neq 0\right\}
$$

и поэтому слои Милнора функций $P / Q$ и $R P /(R Q)$ с $R(0)=0$, вообе говоря, различны.

2. Для $f=P / Q$ пусть $f^{-1}=Q / P$. Нетрудно понять, что $\mathscr{M}_{f^{-1}}^{0}=\mathscr{M}_{f}^{\infty}, \mathrm{a}$ 
$\mathscr{M}_{f-1}^{\infty}=\mathscr{M}_{f}^{0}$. Точно такими же свойствами обладают преобразования монодромии и их дзета-функции, рассматриваемые ниже.

3. Можно (а иногда и более удобно) определять слои Милнора следующим образом:

$$
\begin{aligned}
\mathscr{M}_{f}^{0} & =\left\{z \in B_{\varepsilon}:\|Q(z)\| \leqslant \delta, P(z)=c Q(z) \neq 0\right\}, & & 0<\|c\| \ll \delta \ll \varepsilon, \\
\mathscr{M}_{f}^{\infty} & =\left\{z \in B_{\varepsilon}:\|P(z)\| \leqslant \delta, P(z)=c Q(z) \neq 0\right\}, & & \|c\|^{-1} \ll \delta \ll \varepsilon .
\end{aligned}
$$

Мероморфная функция $f$ определяет отображение из $B_{\varepsilon} \backslash\{P=Q=0\}$ в проективную прямую $\mathbb{C P}^{1}(z \mapsto(P(z): Q(z)))$, которое также будет обозначаться через $f$. Из леммы 1 вытекает, что это отображение является локально тривиальным расслоением над проколотыми окрестностями точек $0=(0: 1)$ и $\infty=(1: 0)$ проективной прямой $\mathbb{C P}^{1}$.

ОПРЕДЕЛЕНИЕ 4. 0-преобразованием монодромии $h_{f}^{0}$ (соответственно $\infty$-преобразованием монодромии $h_{f}^{\infty}$ ) ростка $f$ называется преобразование монодромии расслоения $f$ над петлей $c \cdot \exp (2 \pi i t), t \in[0,1]$, с достаточно малым (соответственно с достаточно большим) $\|c\| \neq 0$.

0 - или $\infty$-оператором монодромии называется действие соответствующего преобразования монодромии в группе гомологий слоя Милнора. Мы хотим применить результаты для мероморфных функций к задаче вычисления дзета-функции многочлена на бесконечности. Поэтому мы будем рассматривать дзета-функции $\zeta_{f}^{0}(t)$ и $\zeta_{f}^{\infty}(t)$ соответствующих преобразований монодромии:

$$
\zeta_{f}^{\bullet}=\prod_{q \geqslant 0}\left\{\operatorname{det}\left[\mathrm{id}-\left.t h_{f *}\right|_{H_{q}\left(\mathscr{M}_{f} ; \mathbb{C}\right)}\right]\right\}^{(-1)^{q}}
$$

$(\cdot=0$ или $\infty)$. Это определение совпадает с используемым в $[3,5]$ и отличается знаком в показателе степени от используемого в [1].

\section{§2. Разрешение особенностей и формула А'Кампо для ростков мероморфных функций}

Пусть $f=P / Q$ - росток мероморфной функции на $\left(\mathbb{C}^{n+1}, 0\right)$, и пусть $\pi: \mathscr{X} \rightarrow \mathscr{U}$ - разрешение ростка $f$. Прообраз $\mathscr{D}=\pi^{-1}(0)$ начала координат в $\mathbb{C}^{n+1}$ является дивизором с нормальными пересечениями. Обозначим через $S_{k, l}$ множество точек дивизора $\mathscr{D}$, в окрестности которых в некоторых локальных координатах поднятия $P \circ \pi$ и $Q \circ \pi$ функций $P$ и $Q$ имеют вид $u y_{0}^{k}$ и $v y_{0}^{l}$ соответственно $(u(0) \neq 0, v(0) \neq 0)$. Небольшая модификация рассуждений А'Кампо [1] позволяет получить следующий вариант его формулы для дзета-функций монодромии мероморфной функции.

ТЕорема 1. Предположим, ито разрешение $\pi: \mathscr{X} \rightarrow \mathscr{U}$ является изоморфизмом вне гиперповерхности $H=\{P=0\} \cup\{Q=0\}$. Тогда

$$
\zeta_{f}^{0}(t)=\prod_{k>l}\left(1-t^{k-l}\right)^{\chi\left(S_{k, l}\right)}, \quad \zeta_{f}^{\infty}(t)=\prod_{k<l}\left(1-t^{l-k}\right)^{\chi\left(S_{k, l}\right)} .
$$


ЗАмечаниЕ. Разрешение $\pi$ ростка $f^{\prime}=R P /(R Q)$ является одновременно разрешением ростка $f=P / Q$. При этом кратности любой компоненты $C$ исключительного дивизора в дивизорах нулей поднятий $(R P) \circ \pi$ и $(R Q) \circ \pi$ ростков $R P$ и $R Q$ получаются из кратностей для ростков $P$ и $Q$ прибавлением одного и того же числа - кратности $m=m(C)$ компоненты $C$ в дивизоре нулей поднятия ростка $R$. Тем не менее, мероморфные функции $f$ и $f^{\prime}$ могут иметь различные дзета-функции. Причина, из-за которой формулы из предыдущей теоремы дают разные результаты для $f$ и для $f^{\prime}$, состоит в том, что если открытая часть компоненты $C$ лежит в $S_{k, l}(f)$, то, вообше говоря, ее часть, которая лежит в $S_{k+m, l+m}\left(f^{\prime}\right)$, меньше.

\section{§3. Дзета-функции мероморфных функций в терминах частичных разрешений}

Пусть $f=P / Q$ - росток мероморфной функции на $\left(\mathbb{C}^{n+1}, 0\right)$, и пусть $\pi:(\mathscr{X}, \mathscr{D}) \rightarrow\left(\mathbb{C}^{n+1}, 0\right)$ - произвольная модификация пространства $\left(\mathbb{C}^{n+1}, 0\right)$, которая является изоморфизмом вне гиперповерхности $H=\{P=0\} \cup\{Q=0\}$ (т.е. $\pi$ не обязательно является разрешением). Пусть $\varphi=f \circ \pi$ - поднятие ростка $f$ на пространство $\mathscr{X}$ модификации, т.е. мероморфная функция $P \circ \pi /(Q \circ \pi)$. Для точки $x \in \pi^{-1}(H)$ пусть $\zeta_{\varphi, x}^{0}(t)$ и $\zeta_{\varphi, x}^{\infty}(t)$ - дзета-функции 0 - и $\infty$-монодромий ростка функции $\varphi$ в точке $x$. Пусть $\mathscr{S}=\{\Xi\}-$ престратификация пространства $\mathscr{D}=\pi^{-1}(0)$ (т. е. его разбиение на полуаналитические подпространства без каких-либо условий регулярности), такая, что для любого страта $\Xi$ из $\mathscr{S}$ дзета-функции $\zeta_{\varphi, x}^{0}(t)$ и $\zeta_{\varphi, x}^{\infty}(t)$ не зависят от $x$ для $x \in \Xi$. Мы обозначим эти дзета-функции через $\zeta_{\Xi}^{0}(t)$ и $\zeta_{\Xi}^{\infty}(t)$ соответственно. Применение конструкций работы [5] в нашей ситуации дает следующее утверждение.

Теорема 2. Для $=0$ или $\infty$

$$
\zeta_{f}^{\bullet}(t)=\prod_{\Xi \in \mathscr{S}}\left[\zeta_{\Xi}(t)\right]^{\chi(\Xi)} .
$$

\section{§4. Дзета-функции в терминах диаграмм Ньютона}

Диаграммой Ньютона $\Gamma=\Gamma(R)$ ростка $R(x)=\sum a_{k} x^{k}$ голоморфной функции $\left(\mathbb{C}^{n+1}, 0\right) \rightarrow(\mathbb{C}, 0)\left(k=\left(k_{0}, k_{1}, \ldots, k_{n}\right), x^{k}=x_{0}^{k_{0}} x_{1}^{k_{1}} \cdot \ldots \cdot x_{n}^{k_{n}}\right)$ называется объединение компактных граней многогранника $\Gamma_{+}=\Gamma_{+}(R)=$ выпуклая оболочка множества $\bigcup_{k: a_{k} \neq 0}\left(k+\mathbb{R}_{+}^{n+1}\right) \subset \mathbb{R}_{+}^{n+1}$.

Пусть $f=P / Q$ - росток мероморфной функции на $\left(\mathbb{C}^{n+1}, 0\right)$, и пусть $\Gamma_{1}=\Gamma(P)$ и $\Gamma_{2}=\Gamma(Q)$ - диаграммы Ньютона ростков $P$ и $Q$. Будем называть пару $\Lambda=\left(\Gamma_{1}, \Gamma_{2}\right)$, состоящую из диаграмм Ньютона $\Gamma_{1}$ и $\Gamma_{2}$, парой Ньютона ростка $f$. Будем говорить, что росток мероморфной функции $f$ невырожден по отношению $\kappa$ своей паре Ньютона $\Lambda=\left(\Gamma_{1}, \Gamma_{2}\right)$, если пара ростков $(P, Q)$ невырожденна по отношению к паре $\Lambda=\left(\Gamma_{1}, \Gamma_{2}\right)$ в соответствии с определением из работы [8] (которое является адаптацией определения А. Г. Хованского из [6] к случаю ростков полных пересечений). 
Определим дзета-функции $\zeta_{\Lambda}^{0}(t)$ и $\zeta_{\Lambda}^{\infty}(t)$ для пары Ньютона $\Lambda=\left(\Gamma_{1}, \Gamma_{2}\right)$. Пусть $1 \leqslant l \leqslant n+1$, и пусть $\mathscr{I}$ - подмножество множества $\{0,1, \ldots, n\}$ с количеством элементов \# $\mathscr{I}$, равным $l$. Пусть $L_{\mathscr{I}}-$ координатное подпространство, $L_{\mathscr{I}}=\left\{k \in \mathbb{R}^{n+1}: k_{i}=0\right.$ для $\left.i \notin \mathscr{I}\right\}, \Gamma_{i, \mathscr{I}}=\Gamma_{i} \cap L_{\mathscr{I}} \subset L_{\mathscr{I}}$. Пусть $L_{\mathscr{I}}^{*}$ - пространство, двойственное к $L_{\mathscr{I}}$, и пусть $L_{\mathscr{I}+}^{*}$ - положительный октант в нем (множество ковекторов, имеющих положительные значения на $L_{\mathscr{I} \geqslant 0}=\left\{k \in L_{\mathscr{I}}: k_{i} \geqslant 0\right.$ для $\left.\left.i \in \mathscr{I}\right\}\right)$. Для примитивного целочисленного ковектора (т.е. неделимого элемента двойственной целочисленной решетки) $a \in\left(\mathbb{R}^{n+1}\right)_{+}^{*}$ положим $m(a, \Gamma)=\min _{x \in \Gamma}(a, x), \Delta(a, \Gamma)=$ $\{x \in \Gamma:(a, x)=m(a, \Gamma)\}$. Обозначим через $m_{\mathscr{I}}$ и $\Delta_{\mathscr{I}}$ соответствующие объекты для диаграммы $Г \mathscr{I}$ и примитивного целочисленного ковектора $a \in L_{\mathscr{I}+}^{*}$. Пусть $E_{\mathscr{I}}-$ множество примитивных целочисленных ковекторов $a \in L_{\mathscr{I}+}^{*}$, для которых $\operatorname{dim}\left(\Delta\left(a, \Gamma_{1}\right)+\Delta\left(a, \Gamma_{2}\right)\right)=l-1$ (суммой Минковского $\Delta_{1}+\Delta_{2}$ двух многогранников $\Delta_{1}$ и $\Delta_{2}$ называется многогранник $\left.\left\{x=x_{1}+x_{2}: x_{1} \in \Delta_{1}, x_{2} \in \Delta_{2}\right\}\right)$. Количество таких ковекторов конечно. Для $a \in E_{\mathscr{I}}$ положим $\Delta_{1}=\Delta\left(a, \Gamma_{1}\right), \Delta_{2}=\Delta\left(a, \Gamma_{2}\right)$, и пусть

$$
V_{a}=\sum_{s=0}^{l-1} V_{l-1}(\underbrace{\Delta_{1}, \ldots, \Delta_{1}}_{s}, \underbrace{\Delta_{2}, \ldots, \Delta_{2}}_{l-1-s}) .
$$

Определение смешанного объема (Минковского) $V\left(\Delta_{1}, \ldots, \Delta_{m}\right)$ см., например, в [4] или в [8]; $(l-1)$-мерный объем в рациональном $(l-1)$-мерном аффинном подпространстве пространства $L_{\mathscr{I}}$ должен быть нормализован таким образом, чтобы объем единичного куба, порожденного любым целочисленным базисом соответствующего линейного подпространства, был равен 1. Напомним, что $V_{m}(\underbrace{\Delta, \ldots, \Delta}_{m})$ - обычный $m$-мерный объем многогранника $\Delta$. Мы должны предполагать, что $V_{0}($ ничего $)=1$ (это необходимо для определения $V_{a}$ при $\left.l=1\right)$. Положим

$$
\begin{aligned}
\zeta_{\mathscr{I}}^{0}(t) & =\prod_{a \in E \mathscr{I}: m\left(a, \Gamma_{1}\right)>m\left(a, \Gamma_{2}\right)}\left(1-t^{m\left(a, \Gamma_{1}\right)-m\left(a, \Gamma_{2}\right)}\right)^{(l-1) ! V_{a}}, \\
\zeta_{\mathscr{I}}^{\infty}(t) & =\prod_{a \in E \mathscr{I}: m\left(a, \Gamma_{1}\right)<m\left(a, \Gamma_{2}\right)}\left(1-t^{m\left(a, \Gamma_{2}\right)-m\left(a, \Gamma_{1}\right)}\right)^{(l-1) ! V_{a}}, \\
\zeta_{l}(t) & =\prod_{\mathscr{I}: \#(\mathscr{I})=l} \zeta_{\mathscr{I}}(t), \quad \zeta_{\Lambda}(t)=\prod_{l=1}^{n+1}\left(\zeta_{l}(t)\right)^{(-1)^{l-1}},
\end{aligned}
$$

где $\cdot=0$ или $\infty$.

ТЕОРема 3. Предположим, что росток $f=P / Q$ мероморфной функиии на $\left(\mathbb{C}^{n+1}, 0\right)$ невырожден по отношению $\kappa$ своей паре Ньютона $\Lambda=$ $\left(\Gamma_{1}, \Gamma_{2}\right)$. Тогда

$$
\zeta_{f}^{0}(t)=\zeta_{\Lambda}^{0}(t), \quad \zeta_{f}^{\infty}(t)=\zeta_{\Lambda}^{\infty}(t) .
$$

ДокАЗАТЕЛЬСтво. Пусть $\Sigma$ - унимодулярное симплициальное разбиение октанта $\mathbb{R}_{\geqslant 0}^{n+1}$, которое соответствует паре диаграмм Ньютона $\left(\Gamma_{1}, \Gamma_{2}\right)$ в 
смысле [8, Sec. 4]. Это разбиение подчинено каждой из диаграмм Ньютона $\Gamma_{1}$ и $\Gamma_{2}$ в смысле работы [9].

Пусть $\pi:(\mathscr{X}, \mathscr{D}) \rightarrow\left(\mathbb{C}^{n+1}, 0\right)$ - торическая модификация, соответствующая $\Sigma$ (см., например, [3]). Поскольку пара $(P, Q)$ невырожденна по отношению к паре диаграмм $\left(\Gamma_{1}, \Gamma_{2}\right), \pi$ является разрешением ростка $f=P / Q$ (см. [8]). Имеем $S_{k, l}=S_{k}(P) \cap S_{l}(Q)$. Описание множеств $S_{k}(P)$ и $S_{l}(Q)$ можно найти в [9, Sec. 7]. Каждое из них состоит из открытых частей некоторых комплексных торов различных размерностей.

Торы размерности $n$ соответствуют положительным (т.е. лежащим в $\left.\left(\mathbb{R}^{n+1}\right)_{+}^{*}\right)$ одномерным конусам в $\Sigma$. Кратность функции $P \circ \pi$ (соответственно функции $Q \circ \pi)$ вдоль такого тора равна $m\left(a, \Gamma_{1}\right)$ (соответственно $\left.m\left(a, \Gamma_{2}\right)\right)$ для примитивного целочисленного ковектора $a$, порождающего соответствующий конус.

Торы размерности $l-1$ соответствуют положительным симплициальным $(n+2-l)$-мерным конусам в $\Sigma$, которые содержат в качестве своей грани конус вида

$$
\mathfrak{S}=\left\{a \in\left(\mathbb{R}^{n+1}\right)_{\geqslant 0}^{*}: a_{j}>0 \text { для } j \notin \mathscr{I}, a_{j}=0 \text { для } j \in \mathscr{I}\right\}
$$

с $\#(\mathscr{I})=l$ (эти грани сами являются элементами разбиения $\Sigma)$. Эти конусы, в свою очередь, соответствуют одномерным конусам разбиения октанта $L_{\mathscr{I} \geqslant 0}$, подчиненного диаграмме Ньютона $\Gamma_{i, \mathscr{I}}=\Gamma_{i} \cap L_{\mathscr{I}} \subset L_{\mathscr{I}}$. Кратности функций $P \circ \pi$ и $Q \circ \pi$ вдоль такого тора равны $m_{\mathscr{I}}\left(a, \Gamma_{1, \mathscr{I}}\right)$ и $m_{\mathscr{I}}\left(a, \Gamma_{2, \mathscr{I}}\right)$ соответственно, где $a$ - примитивный целочисленный ковектор, порождающий соответствующий одномерный конус.

Для того чтобы применить теорему 1, нужно вычислить эйлерову характеристику соответствующей части $(l-1)$-мерного тора $T$ - дополнения к пересечению с собственным прообразом гиперповерхности $H=\{P=0\} \cup\{Q=0\}$. Пусть $A$ (соответственно $B)$ - пересечение тора $T$ с собственным прообразом гиперповерхности $\{P=0\}$ (соответственно $\{Q=0\}$ ). Положим $\Delta_{i}:=$ $\Delta\left(a, \Gamma_{i, \mathscr{I}}\right)$. Из результатов Хованского [7] следует, что эйлерова характеристика гиперповерхности $A$ (соответственно $B$ ) равна

$$
(-1)^{l}(l-1) ! V_{l-1}(\underbrace{\Delta_{1}, \ldots, \Delta_{1}}_{l-1})\left(\text { соответственно }(-1)^{l}(l-1) ! V_{l-1}(\underbrace{\Delta_{2}, \ldots, \Delta_{2}}_{l-1})\right),
$$

а эйлерова характеристика гиперповерхности $A \cap B$ равна

$$
\begin{array}{r}
(-1)^{l-1}(l-1) !\left[V_{l-1}(\underbrace{\Delta_{1}, \ldots, \Delta_{1}}_{l-2}, \Delta_{2})+V_{l-1}(\underbrace{\Delta_{1}, \ldots, \Delta_{1}}_{l-3}, \Delta_{2}, \Delta_{2})+\cdots\right. \\
\left.+V_{l-1}(\Delta_{1}, \underbrace{\Delta_{2}, \ldots, \Delta_{2}}_{l-2})\right] .
\end{array}
$$


Таким образом, эйлерова характеристика дополнения к $A \cup B$ в торе $T$ равна

$$
\begin{aligned}
& \begin{aligned}
\chi(T) & -\chi(A)-\chi(B)+\chi(A \cap B) \\
= & (-1)^{l-1}(l-1) !\left[V_{l-1}(\underbrace{\Delta_{1}, \ldots, \Delta_{1}}_{l-1})+V_{l-1}(\underbrace{\Delta_{1}, \ldots, \Delta_{1}}_{l-2}, \Delta_{2})+\cdots\right. \\
& \left.+V_{l-1}(\underbrace{\Delta_{2}, \ldots, \Delta_{2}}_{l-1})\right],
\end{aligned} \\
& \text { что и доказывает утверждение. } \square
\end{aligned}
$$

\section{§5. Формула типа Варченко для $f=P / z_{0}^{d}$}

Как указывалось в начале статьи, для изучения поведения многочленов на бесконечности представляют интерес ростки мероморфных функций вида $P\left(z_{0}, z_{1}, \ldots, z_{n}\right) / z_{0}^{d}$. В этом случае формулы для дзета-функций $\zeta_{\Lambda}^{0}(t)$ и $\zeta_{\Lambda}^{\infty}(t)$ существенно упрощаются. Переформулируем определение этих дзетафункций для случая, когда диаграмма Ньютона $\Gamma_{2}$ состоит из одной точки $(d, 0, \ldots, 0)$ (в терминах диаграммы Ньютона $\Gamma:=\Gamma_{1}$ ростка $P$ ). Получаем следующее описание.

Пусть $1 \leqslant l \leqslant n+1$, и пусть $\mathscr{I}$ - подмножество множества $\{1, \ldots, n\}$ с количеством элементов \# $\mathscr{I}$, равным $l-1$. Пусть $\gamma_{1}^{\mathscr{I}}, \ldots, \gamma_{j(\mathscr{I})}^{\mathscr{\mathscr { T }}}-$ все $(l-1)$-мерные грани диаграммы $\Gamma_{\mathscr{I} \cup\{0\}}, a_{\mathscr{I}, 1}, \ldots, a_{\mathscr{I}, j(\mathscr{I})}$ - соответствующие примитивные ковекторы (ортогональные к $\left.\gamma_{1}^{\mathscr{I}}, \ldots, \gamma_{j(\mathscr{I})}^{\mathscr{I}}\right), a_{\mathscr{I}, s}^{0}$ - нулевая координата ковектора $a_{\mathscr{I}, s}$, и пусть $m_{s}(\mathscr{I})=\left(a_{\mathscr{I}, s}, k\right)$ для $k \in \gamma_{s}^{\mathscr{I}}$. Тогда

$$
\begin{aligned}
\zeta_{\mathscr{I} \cup\{0\}}^{0}(t) & =\prod_{1 \leqslant s \leqslant j(\mathscr{I}): m_{s}(\mathscr{I})>d \cdot a_{\mathscr{I}, s}^{0}}\left(1-t^{m_{s}(\mathscr{I})-d \cdot a_{\mathscr{I}, s}^{0}}\right)^{(l-1) ! V_{l-1}\left(\gamma_{s}^{\mathscr{I}}\right)}, \\
\zeta_{\mathscr{I} \cup\{0\}}^{\infty}(t) & =\prod_{1 \leqslant s \leqslant j(\mathscr{I}): m_{s}(\mathscr{I})<d \cdot a_{\mathscr{I}, s}^{0}}\left(1-t^{d \cdot a_{\mathscr{I}, s}^{0}-m_{s}(\mathscr{I})}\right)^{(l-1) ! V_{l-1}\left(\gamma_{s}^{\mathscr{I}}\right)}, \\
\zeta_{l}(t) & =\prod_{\mathscr{I} \subset\{1, \ldots, n\}: \# \mathscr{I}=l-1} \zeta_{\mathscr{I} \cup\{0\}}^{\dot{I}^{\prime}}(t), \quad \zeta_{\Lambda}(t)=\prod_{l=1}^{n+1}\left(\zeta_{l}^{\dot{I}^{\prime}}(t)\right)^{(-1)^{l-1}}
\end{aligned}
$$

$(\bullet=0$ или $\infty)$, где $V_{l-1}\left(\gamma_{s}^{\mathscr{I}}\right)-($ обычный $)(l-1)$-мерный объем грани $\gamma_{s}^{\mathscr{I}}$ (в гиперплоскости в $L_{\mathscr{I} \cup\{0\}}$, порожденной этой гранью).

\section{§6. Примеры}

ПримеР 1. Пусть $f=\left(x^{3}-x y\right) / y$. Слой Милнора $\mathscr{M}_{f}^{0}$ (соответственно $\left.\mathscr{M}_{f}^{\infty}\right)$ равен $\left\{(x, y):\|(x, y)\|<\varepsilon,\left(x^{3}-x y, y\right) \in B_{\delta}, x^{3}-x y=c y\right\} \backslash\{(0,0)\}$, где $\|c\| \neq 0$ достаточно мало (соответственно достаточно велико). Из уравнения $x^{3}-x y=c y$ получаем $y=x^{3} /(x+c)$, и поэтому слой Милнора $\mathscr{M}_{f}^{0}$ диффеоморфен диску $\mathscr{D}$ на $x$-плоскости без двух точек: $-c$ и нуля. Точно так же слой Милнора $\mathscr{M}_{f}^{\infty}$ диффеоморфен проколотому диску $\mathscr{D}^{*}$. Нетрудно понять, что в обоих случаях действие преобразования монодромии в группах 
гомологий тривиально. Таким образом

$$
\zeta_{f}^{0}(t)=(1-t)^{-1}, \quad \zeta_{f}^{\infty}(t)=1 .
$$

Вычислим эти дзета-функции через диаграммы Ньютона (рис. 1).

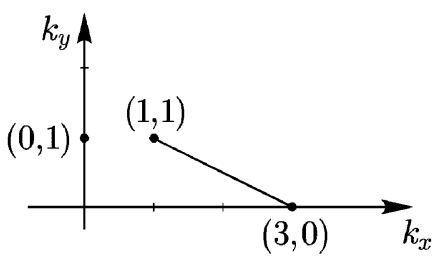

Рис. 1

Поскольку каждая координатная ось пересекается только с одной из диаграмм, $\zeta_{1}^{\bullet}(t)=1$. Имеется только одна линейная функция (а именно, $a=$ $\left.k_{x}+2 k_{y}\right)$, для которой $\operatorname{dim} \Delta\left(a, \Gamma_{1}\right)=1$. Одномерный объем $V_{1}\left(\Delta\left(a, \Gamma_{1}\right)\right)$ грани $\Delta\left(a, \Gamma_{1}\right)$ равен $1, V_{1}\left(\Delta\left(a, \Gamma_{2}\right)\right)=0$. Имеем $m\left(a, \Gamma_{1}\right)=3, m\left(a, \Gamma_{2}\right)=2$. Таким образом, $\zeta_{2}^{0}(t)=1-t, \zeta_{2}^{\infty}(t)=1, \zeta_{\left(\Gamma_{1}, \Gamma_{2}\right)}^{0}(t)=(1-t)^{-1}, \zeta_{\left(\Gamma_{1}, \Gamma_{2}\right)}^{\infty}(t)=1$, что совпадает с написанными выше формулами.

ПримеР 2. Пусть $P=x y z+x^{p}+y^{q}+z^{r}$ - особенность $T_{p, q, r}, 1 / p+$ $1 / q+1 / r<1$, и пусть $Q=x^{d}+y^{d}+z^{d}$ - однородный многочлен степени $d$. Предположим, что $p>q>r>d>3$ и что $p, q$ и $r$ попарно взаимно просты. Вычислим дзета-функции монодромий ростка $f=P / Q$ с помощью теорем 2 и 3.

(a) Очевидно, что росток $f$ невырожден по отношению к своей паре Ньютона $\Lambda=\left(\Gamma_{1}, \Gamma_{2}\right)$. Поэтому

$$
\zeta_{f}(t)=\zeta_{\Lambda}(t)=\zeta_{1}\left(\zeta_{2}^{\bullet}\right)^{-1} \zeta_{3} \quad(\bullet=0 \text { или } \infty) .
$$

Имеем $\zeta_{1}^{\infty}=\zeta_{2}^{\infty}=1$, и единственный ковектор, необходимый для вычисления $\zeta_{3}^{\infty}$, 一 это $a=(1,1,1)$. В этом случае $m\left(a, \Gamma_{1}\right)=3, m\left(a, \Gamma_{2}\right)=d, \Delta\left(a, \Gamma_{1}\right)=$ $\{(1,1,1)\}$, а $\Delta\left(a, \Gamma_{2}\right)$ - симплекс $\left\{k_{x}+k_{y}+k_{z}=d, k_{x} \geqslant 0, k_{y} \geqslant 0, k_{z} \geqslant 0\right\}$, его двумерный объем равен $d^{2} / 2$. Таким образом, $\zeta_{f}^{\infty}=\left(1-t^{d-3}\right)^{d^{2}}$.

Имеем

$$
\begin{gathered}
\zeta_{1}^{0}=\left(1-t^{p-d}\right)\left(1-t^{q-d}\right)\left(1-t^{r-d}\right), \\
\zeta_{2}^{0}=\left(1-t^{r(q-d)}\right)\left(1-t^{r(p-d)}\right)\left(1-t^{q(p-d)}\right)\left(1-t^{r-d}\right)^{2 d}\left(1-t^{q-d}\right)^{d} .
\end{gathered}
$$

Чтобы вычислить $\zeta_{3}^{0}$, нужно учитывать как ковекторы $(r q-q-r, r, q)$, $(r, p r-p-r, p)$ и $(q, p, q p-p-q)$, соответствующие двумерным граням диаграммы $\Gamma_{1}$, так и ковекторы $(1, r-2,1),(r-2,1,1)$ и $(q-2,1,1)$, соответствующие парам вида (одномерная грань диаграммы $\Gamma_{1}$, одномерная грань диаграммы $\left.\Gamma_{2}\right)$. Например, для $a=(1, r-2,1)$ грань $\Delta\left(a, \Gamma_{1}\right)$ (соответственно грань $\left.\Delta\left(a, \Gamma_{2}\right)\right)$ - отрезок, соединяющий точки $(0,0, r)$ и $(1,1,1)$ 
(соответственно соединяющий точки $(d, 0,0)$ и $(0,0, d))$. Обратите внимание на «отсутствие симметрии»: последние три ковектора не получаются друг из друга перестановкой координат и чисел $p, q$ и $r$. Таким образом,

$$
\begin{gathered}
\zeta_{3}^{0}=\left(1-t^{r(q-d)}\right)\left(1-t^{r(p-d)}\right)\left(1-t^{q(p-d)}\right)\left(1-t^{r-d}\right)^{2 d}\left(1-t^{q-d}\right)^{d}, \\
\zeta_{f}^{0}=\left(1-t^{p-d}\right)\left(1-t^{q-d}\right)\left(1-t^{r-d}\right) .
\end{gathered}
$$

(b) Для вычисления дзета-функций ростка $f$ с помощью теоремы 2 в качестве модификации $\pi:(\mathscr{X}, \mathscr{D}) \rightarrow\left(\mathbb{C}^{3}, 0\right)$ возьмем раздутие $(\sigma$-процесс) начала координат в $\mathbb{C}^{3}$, и пусть $\varphi$ - поднятие $f \circ \pi$ ростка $f$ на пространство $\mathscr{X}$. Исключительным дивизором $\mathscr{D}$ модификации является комплексная проективная плоскость $\mathbb{C P}^{2}$. Пусть $H_{1}$ и $H_{2}$ - собственные прообразы гиперповерхностей $\{P=0\}$ и $\{Q=0\}$ и $D_{i}=\mathscr{D} \cap H_{i}$. Кривая $D_{1}$ состоит из трех трансверсальных прямых $l_{1}, l_{2}$ и $l_{3}$ и имеет три особые точки $S_{1}=l_{2} \cap l_{3}=(0,0,1), S_{2}=l_{1} \cap l_{3}=(0,1,0)$ и $S_{3}=l_{1} \cap l_{2}=(1,0,0)$. Кривая $D_{2}$ - это неособая кривая степени $d$. Она пересекает $D_{1}$ трансверсально в $3 d$ различных неособых точках $\left\{P_{1}, \ldots, P_{3 d}\right\}$.

Имеется следуюшая естественная стратификация исключительного дивизора $\mathscr{D}$ :

(i) нульмерные страты $\Lambda_{i}^{0}(i=1,2,3)$, каждый состоящий из одной точки $S_{i}$

(ii) нульмерные страты $\Xi_{i}^{0}$, каждый состоящий из одной точки $P_{i}(i=$ $1, \ldots, 3 d)$

(iii) одномерные страты $\Xi_{i}^{1}=l_{i} \backslash\left\{D_{2} \cup l_{j} \cup l_{k}\right\}(i=1,2,3)$ и $\Xi_{4}^{1}=D_{2} \backslash D_{1}$;

(iv) двумерный страт $\Xi^{2}=\mathscr{D} \backslash\left(D_{1} \cup D_{2}\right)$.

Нетрудно видеть, что $\zeta_{\Xi_{2}}^{0}(t)=1, \zeta_{\Xi_{2}}^{\infty}(t)=1-t^{d-3}$ и для каждого страта $\Xi$ из $\Xi_{i}^{0}(1 \leqslant i \leqslant 3 d)$ или из $\Xi_{i}^{1}(1 \leqslant i \leqslant 4)$ имеем $\zeta_{\Xi}^{\bullet}(t)=1(\cdot=0$ или $\infty)$.

Далее предполагается, что исключительный дивизор $\mathscr{D}$ локально задается уравнением $u=0$. В точке $S_{1}$ росток поднятия $\varphi$ функции $f$ имеет вид $\left(u^{3} x_{1} y_{1}+u^{r}+x_{1}^{p} u^{p}+y_{1}^{q} u^{q}\right) /\left(u^{d} x_{1}^{d}+u^{d} y_{1}^{d}+u^{d}\right)$. Этот росток имеет ту же пару Ньютона, что и росток $\left(u^{3} x_{1} y_{1}+u^{r}\right) / u^{d}$. Пользуясь теоремой 3 , получаем $\zeta_{\Lambda_{1}^{0}}^{\infty}=1, \zeta_{\Lambda_{1}^{0}}^{\infty}=1-t^{r-d}$. В точке $S_{2}$ росток функции $\varphi$ имеет вид $\left(u^{3} x_{1} z_{1}+z_{1}^{r} u^{r}+x_{1}^{p} u^{p}+u^{q}\right) /\left(u^{d} x_{1}^{d}+u^{d}+z_{1}^{d} u^{d}\right)$. Он имеет ту же пару Ньютона, что и $\left(u^{3} x_{1} z_{1}+z_{1}^{r} u^{r}+u^{q}\right) / u^{d}$. Из теоремы 3 следует, что $\zeta_{\Lambda_{2}^{0}}^{\infty}(t)=1$, $\zeta_{\Lambda_{2}^{0}}^{0}(t)=1-t^{q-d}$. Точно так же $\zeta_{\Lambda_{3}^{0}}^{\infty}(t)=1, \zeta_{\Lambda_{3}^{0}}^{0}(t)=1-t^{p-d}$. Собирая вместе эти вычисления, получаем тот же результат, что и выше (без использования частичного разрешения).

\section{ЛитературА}

1. A'Campo N. La fonction zêta d'une monodromie. Comm. Math. Helv., 50, 233-248 (1975).

2. Арнольд В. И. Особенности дробей и поведение многочленов на бесконечности, Труды МИРАН, 221 (1998) (в печати). 
3. Арнольд В. И., Варченко А. Н., Гусейн-Заде С. М. Особенности дифференцируемых отображений, т. 2. Наука, М., 1984.

4. Бернитейн Д. Н. Число корней системы уравнений. Функц. анализ и его прил., 9, вып. 3, 1-4 (1975).

5. Gusein-Zade S. M., Luengo I., Melle-Hernández A. Partial resolutions and the zetafunction of a singularity. Comm. Math. Helv., 72, 244-256 (1997).

6. Хованский А. Г. Многогранники Ньютона и торические многообразия. Функц. анализ и его прил., 11, вып. 4, 56-67 (1977).

7. Хованский A. Г. Многогранники Ньютона и род полных пересечений. Функц. анализ и его прил., 12, вып. 1, 51-61 (1978).

8. Oka $M$. Principal zeta-function of non-degenerate complete intersection singularity. J. Fac. Sci. Univ. Tokyo Sect. IA Math., 37, 11-32 (1990).

9. Varchenko A. N. Zeta function of monodromy and Newton's diagram. Invent. Math., 37, 253-262 (1976).

Московский государственный университет механико-математический факультет

Поступило в редакцию

e-mail: sabir@ium.ips.ras.ru

3 июля 1997 г.

Facultad de Ciencias Matemáticas,

Universidad Complutense de Madrid.

e-mail: iluengo@eucmos.sim.ucm.es

Facultad de Ciencias Matemáticas, Universidad Complutense de Madrid. e-mail: amelle@eucmos.sim.ucm.es 